\title{
Challenging Saccular Aorto-iliac Aneurysms in Behcet's Disease
}

\author{
Hussien M Rabee ${ }^{1 *}$, Khalid A Brekeit ${ }^{2}$, Adham Al-Bakry ${ }^{3}$ and Sameh Dimitri ${ }^{4}$ \\ ${ }^{1}$ Department of Vascular Surgery, Countess of Chester Hospital, Chester, UK \\ ${ }^{2}$ Department of Surgery, College of Medicine, Al-Imam Muhammad Ibn Saud Islamic University, Saudi Arabia \\ ${ }^{3}$ Department of Renal Transplant Surgeon, Royal Liverpool University Hospital, UK \\ ${ }^{4}$ Department of Vascular Surgery, Countess of Chester Hospital, Chester, UK
}

*Corresponding author: Hussien M Rabee, Department of Vascular Surgery, Countess of Chester Hospital, NHS Foundation trust, South Merseyside Arterial Centre, CH2 1UL, Chester, UK.

Received Date: December 05, 2018

Published Date: December 14, 2018

\begin{abstract}
Background: Behect's disease is a systemic vasculitic disease with uncertain aetiology and affecting wide range of blood vessel sizes in young age group of patients. Vascular involvement in Behcet's varies in the literature from 7 to $34 \%$. Arterial lesions in Behect's disease is very uncommon and carries a relatively high rate of mortality when rupture due to involvement of pulmonary or aorto-iliac arteries.
\end{abstract}

Methods: This is a retrospective review of nine patients with Behcet's disease (BD) and saccular aorto-iliac aneurysms. All of them treated surgically. Six patients presented with aortic saccular aneurysms and 3 involved the common iliac artery. Open surgical repair was performed to 6 patients, 2 patients had endovascular treatment and 1 patient had both (hybrid procedure).

Result: Initial success of aneurysmal treatment was achieved in 8 cases. Two patients developed small false aneurysms at the arterial puncture sites. There is 1 case of operative mortality. Two cases developed recurrence at the anastomotic line. Three patients died in less than 18 months follow-up.

Conclusion: Arterial lesions of Behect's disease are rare conditions with high potential risk of complications. Endovascular management might potentially result in better outcome.

\section{Introduction}

Behcet's disease (BD) is a known multisystem vasculitis of unknown aetiology and rarely affecting arterial system with aneurysms [1]. It is a real challenge to vascular surgeons as they need to treat the disease as well as dealing with the aneurysms [1]. Aneurysm is generally defined as abnormal local dilatation of a blood vessel more than $50 \%$ or $3 \mathrm{~cm}$ of the transverse diameter $[2,3]$. It is considered as wall weakening and a significant fatal risk of rupture when it exceeds $5.5 \mathrm{~cm}$ [4]. Majority of is aneurysms are arterial and to a lower extent is demonstrated in venous wall or cardiac chambers. Smoking is the commonest associate risk factors [5] and there is growing evidences that diabetes is protective against aneurysmal dilatation [6]. Atherosclerosis is the main aetiological type of aneurysm and usually fusiform in shape [3]. This morphology reflects an even distribution of the aneurysmal pathogenesis within the arterial wall with connective tissue matrix degradation [7]. Also, Non-atherosclerotic aneurysmal aetiologies have been identified including traumatic, mycotic and vasculitic aneurysms [8,9]. In which, abnormal leucocytic activities occlude the vasa vasorum inducing endarteritis obliterans and causing a focal necrosis of the arterial wall. This might progress to perforation or localized weakness with subsequent dilatation and false aneurysm formation [10]. In such condition, a saccular aneurysm is the presenting morphology of the aneurysm [8]. We are presenting our experience in management of challenging saccular aorto-iliac aneurysms in patients with Behcet's disease (BD). Patients were consented for presentation and publication.

\section{Patients \& Methods}

This is a retrospective review of nine patients with Behcet's disease (BD) and saccular aorto-iliac aneurysms. They are managed and surgically treated in two vascular centres: 1) King Khalid 
University Hospital and 2) Security Forces Hospital 3) Military Forces Hospital, Riyadh, Saudi Arabia from January 1995 till April 2014. All patients presented with classical Behcet's triad of oral and genital ulcers with uveitis [11]. The age ranged from 21 to 43 years with median age of 29 years. Seven patients were males. Two patients were smokers and one was diabetic. Five patients had infra-renal abdominal aortic aneurysms AAA, one had type IV thoraco-abdominal aortic aneurysm TAAA and 3 had right common iliac artery CIA aneurysm (Table 1). Six patients had elective interference and 3 were treated as emergency cases. All patients had CT angiogram. Direct arteriogram study was performed in 3 cases and MRI in 7patients. Six patients were treated with open surgical procedures, 2 had endovascular exclusion and one patient had hybrid (both) procedures (Table 2). The elective received medical therapy prior to the interference in order to control active state of the disease.

\section{Result}

In Six patients, arterial aneurysms were incidentally discovered during scanning for other unrelated condition and 3 presented with leaking ruptured aneurysm. Six patients had associated neurological manifestations and 4 had venous thrombosis; 3 cases of DVT and one case of Inferior vena caval obstruction complicating to Budd-Chiari syndrome (Table1). All iliac aneurysms were at the right side. The transvers diameter of the aneurysms is shown in Table 2. Three patients had multiple smaller aneurysms and treated consevatively. Out of the seven surgically treated, 5 had aneurysmal replacement with synthetic grafts (4 tubal and 1 bifurcated) and 2 patient had patch angioplasty at the small aortic wall defect. An aortic occluding balloon was used intra-luminally to block the blood flow prior to opening the aneurysmal sac and suturing the patch at the aortic defect (Figure 1-3). Hybrid procedures consisted of endovascular exclusion of juxta-renal aneurysm with non-functioning right kidney with revascularization of both left renal and superior mesenteric artery via an inverted bifurcated graft from left common iliac artery. Post-operative CT angiogram confirmed successful aneurysm exclusion and patency of ilio-renal-mesenteric bypass (Figures $4 \& 5$ ). There was one operative mortality (within 30 days) which is the patient with type IV Thoraco-abdominal aneurysm. The patient died secondary to right side massive haemothorax as a complication of central line insertion. Histopathological studies of the sac wall were done in 4 cases confirmed a diagnosis of vasculitis, with perivascular granulomatous reaction destroying the media and dense infiltration with lymphocytes. Two patients developed small false aneurysm at the arterial puncture sites (common femoral arteries) and treated conservatively. Follow-up with ultrasound and/or CT angiography ranged from 6 to 47 months (median is 17 months). Recurrence of aneurysm at the anastomotic suture line was detected in 2 cases at 11- and 23-months interval. Two patients were lost from follow up in approximately 9 months' time and 3 patients died within 18 months from the surgical procedures.

Table 1: Presentations of Behcet disease patients with aorto-iliac saccular aneurysms.

\begin{tabular}{|c|c|c|c|c|c|c|}
\hline No. & Age y & Gender & Site & Risk Factors & Presentation & Associated lesions \\
\hline 1 & 26 & Female & AAA & Smoker & Rupture & IVC obstruction \\
\hline 2 & 32 & Male & AAA & - & Elective & DVT + Neurological \\
\hline 3 & 29 & Male & AAA & Diabetes & Rupture & DVT \\
\hline 4 & 43 & Female & Iliac & - & Elective & Neurological \\
\hline 5 & 34 & Male & AAA & - & Elective & Neurological \\
\hline 6 & 30 & Male & TAAA & Smoker & Elective & DVT \\
\hline 7 & 21 & Male & AAA & - & Elective & Neurological \\
\hline 8 & 25 & Male & Iliac & - & Rupture & Neurological \\
\hline 9 & 28 & Male & Iliac & - & Elective & Neurological \\
\hline
\end{tabular}

Table 2: Summary of the diagnostic tests and surgical procedures.

\begin{tabular}{|c|c|c|c|c|c|c|}
\hline Case no. & $\begin{array}{c}\text { Transverse } \\
\text { Diameter cm. }\end{array}$ & CT angiogram & Arteriogram & MRI & Surgical & Endovascular \\
\hline 1 & 7 & + & - & - & $\mathrm{Y}$ & - \\
\hline 2 & 5.5 & + & + & - & Y & - \\
\hline 3 & 6.2 & + & - & + & $\mathrm{Y}$ & - \\
\hline 4 & 3.7 & + & + & + & - & $\mathrm{Y}$ \\
\hline 5 & 5.7 & + & + & + & $\mathrm{Y}$ & $\mathrm{Y}$ \\
\hline 6 & 6 & + & - & + & $\mathrm{Y}$ & - \\
\hline 7 & 14.2 & + & - & + & $\mathrm{Y}$ & - \\
\hline 8 & 4.1 & + & - & + & $\mathrm{Y}$ & - \\
\hline 9 & 3.6 & + & - & + & - & $\mathrm{Y}$ \\
\hline
\end{tabular}




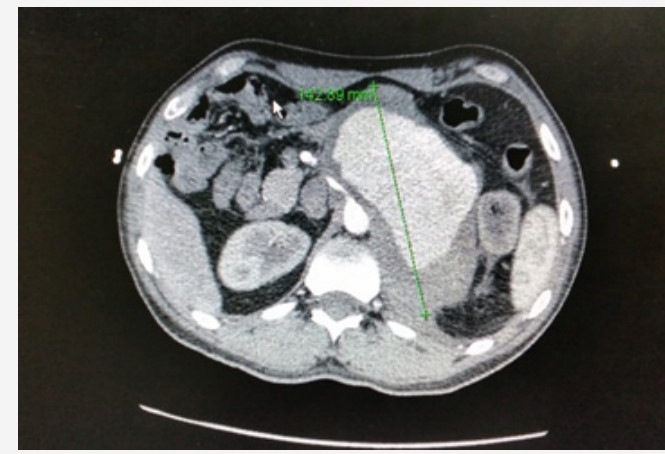

Figure 1: Leaking huge saccular abdominal aortic aneurysm AAA.

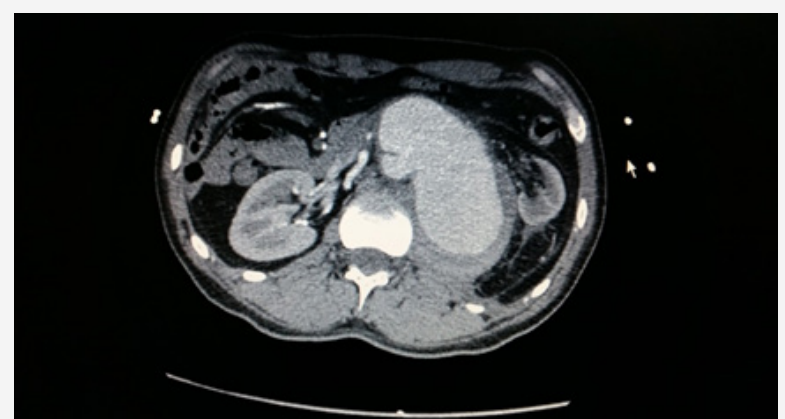

Figure 2: Large saccular infra-renal abdominal aortic aneurysm in patient with Behcet Disease. The arrow is pointing to a narrow neck at aortic origin.

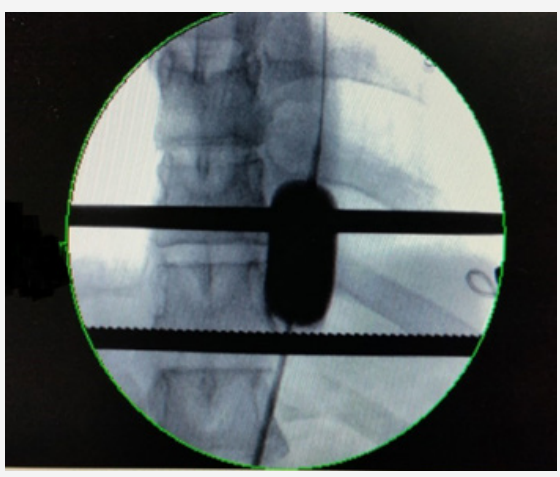

Figure 3: An aortic occluding balloon was used intra-luminally to block the blood flow prior to opening the aneurysmal sac and suturing the patch at the aortic defect.
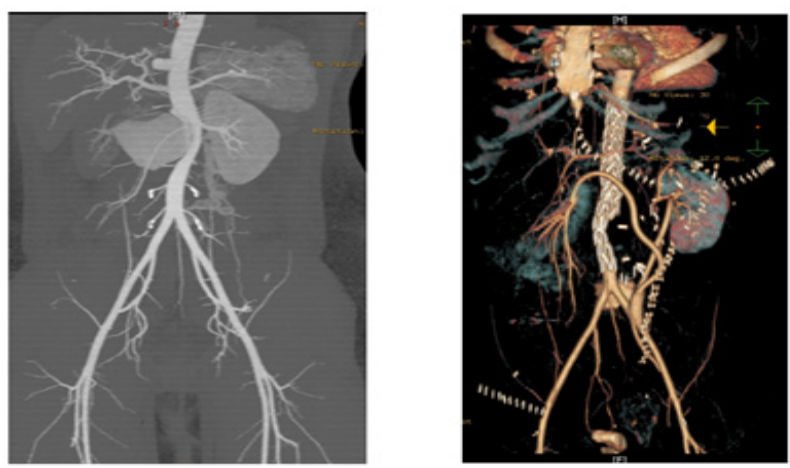

Figure 4: CT angio. of pre and post hybrid procedure consisted of endovascular exclusion of juxta-renal aneurysm with nonfunctioning right kidney with revascularization of both left renal and superior mesenteric artery via an inverted bifurcated graft from left common iliac artery.

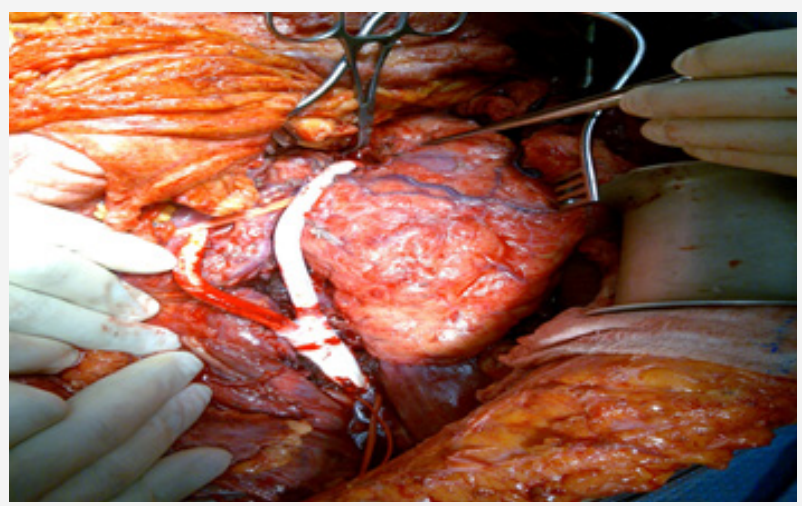

Figure 5: Intra-operative imaging of the inverted bifurcated synthetic graft bypass from left common iliac artery to left renal and superior mesenteric arteries.

Behect's disease is a systemic vasculitic disease with uncertain aetiology and affecting wide range of blood vessel sizes in young age group of patients [9]. It was reported in a jejunal artery presented with intra-abdominal bleeding [12]. It is more common in middle east and far east areas [9]. Reports from Saudi Arabia, Turkey, and Iraq demonstrate the presence of histocompatibility antigen HLA B5 (51) in approximately $72 \%$ of patients with Behcet's disease. These results have not been confirmed by studies conducted in the United Kingdom [13]. A familial pattern of disease has been described, but whether this pattern is due to true genetic predisposition or exposure to a common precipitating agent in immunogenetically susceptible individuals is unclear [14]. It is generally agreed that immunosuppressant therapy is advantageous during the active stage and controlling the complications of vasculitis in Behcet's disease [15]). The choice of immunosuppressive therapy in severe disease such as uveitis and arteritis is between alkylating agents and agents that inhibit interleukin-2 production [16,17]. I this study, all cases are aorto-iliac and not visceral. These cases were referred to us as tertiary centres and we followed the same protocol od pre-operative medical therapy to control active state of Behcet's disease. We recommend this protocol to avoid facing friable arterial tissue adjacent to the aneurysmal are during active phase of the disease.

The vascular involvement in Behcet's varied in the literature from 7 to $34 \%$ [18]. Association with deficiencies of naturally circulating anticoagulants such as protein C, protein S and factor VIII are causing higher incidence of venous thrombosis and indicates long term anticoagulation [19-21]. In this study, two thirds of the patients found to have venous thrombosis. Arterial lesions in Behect's disease is very uncommon and carries a relatively high rate of mortality when rupture due to involvement of pulmonary or aorto-iliac arteries [22]. This series demonstrate the cases referred to our vascular surgery units while other thoracic cases are referred to related specialities.

CT angio. and MRA became the diagnostic tests to diagnose and plan of surgical modalities. Arteriogram is considerted contraindicated as it is having a high risk of developing thrombosis, bleeding and false aneurysm at the puncture site. In our series, we get false aneurysm in the 2 cases out of three had direct arteriogram done early in the study $[10,11]$. 
Surgical treatment is associated with high risk of thrombotic complications, recurrence and anastomotic leak and several retrospective analysis and cases reports are discouraging $[9,23]$. The mortality case in this series demonstrated of a potential risk of arterial complication during performing invasive procedures in patients with Behcet's disease. Unfortunately, post mortem autopsy was not available to figure out the source of this massive bleeding. Postoperative anti-coagulation, steroids and chemotherapy postulated to improve the survival [17]. However, 25\% incidence of recurrence is reported [24]. Ligation of major vessels were reported to avoid the fatal recurrence and bleeding complications [25]. Some surgeons believe that venous conduits should be preferable than synthetics. In our series, none of the conduits was venous. The critical arterial diameter to indicate surgical treatment of the aneurysms before rupturing is not defined. Unfortunately, physicians are not routinely scanning patients with Behect's disease for potential arterial involvement. Therefore, arterial aneurysm presents late and diagnosed with complications.

Central nervous system (CNS) involvement occurs in a variable proportion of cases [26]. Six of our patients had concomitant neurological involvement. In our study, we used to scan those patients annually. Hybrid technique of endovascular exclusion and surgical bypass for essential visceral aortic branches has been replaced by fenestrated and branched EVAR techniques. Recently, the endovascular management showed better results and less morbidity [23]. We declare that longer follow-up was not available in this series especially for female patients.

\section{Conclusion}

Arterial lesions of Behect's disease are rare conditions with high potential risk of complications. We recommend centralization of those patients to augment the surgical experience and empower the research.

management might potentially result in better outcome but long-term follow-up as well as additional solid evidences are highly needed.

\section{Acknowledgement}

None.

\section{Conflict of Interest}

No conflict of interest.

\section{References}

1. Enoch BA, Castillo-Olivares JL, Khoo TC, Grainger RG, Henry L (1968) Major vascular complications in Behçet's syndrome. Postgrad Med J 44(512): 453-459.

2. Lederle FA, Johnson GR, Wilson SE, Chute EP, Littooy FN, et al. (1997) Prevalence and associations of abdominal aortic aneurysm detected through screening. Aneurysm Detection and Management (ADAM) Veterans Affairs Cooperative Study Group. Ann Int Med 126(6): 441-449.

3. Sakalihasan N, Limet R, Defawe OD (2005) Abdominal aortic aneurysm. Lancet 365(9470): 1577-1589.

4. Maxon V, Parr A, Emeto TI, Walker P, Norman PE, et al. (2010) Diagnosis and monitoring of abdominal aortic aneurysm: Current status and future prospects. Curr Probl Cardiol 35(10): 512-548.

5. Norman PE, Curci JA (2013) Understanding the effects of tobacco smoke on the pathogenesis of aortic aneurysm. Arterioscler Thromb Vasc Biol 33(7): 1473-1477.

6. Raffort J, Lareyre F, Clément M, Hassen-Khodja R, Chinetti G, et al. (2018) Diabetes and aortic aneurysm: current state of the art. Cardiovasc Res 114(13): 1702-1713.

7. Amato B, Compagna R, Amato M, Grande R, Butrico L, et al. (2015) Adult vascular wall resident multipotent vascular stem cells, matrix metalloproteinases, and arterial aneurysms. Stem Cells Int 2015: 434962.

8. Panayiotopoulos YP, Assadourian R, Taylor PR (1996) Aneurysms of the visceral and renal arteries. Ann R Coll Surg Engl 78(5): 412-419.

9. Rabee H, Al-Saleh M, Al-Salman M (1999) Ruptured Abdominal Aortic Aneurysm and Inferior Vena Cava Occlusion in Behqet's Disease, A Case Report. J Vasc Surg 33(3): 323-328.

10. Park JH, Ham MC, Bettman MA (1984) Arterial manifestations of Behqet's disease. AJR Am J Roentgenol 143: 821-825.

11. Kalra S, Silman A, Akman-Demir G, Bohlega S, Borhani-Haghighi A, et al. (2013) Diagnosis and management of Neuro-Behçet's disease: international consensus recommendations. J Neurol 261(9): 1662-1676.

12. Wu XY, Wei JP, Zhao XY, Wang Y, Wu HH, et al. (2015) Spontaneous IntraAbdominal Hemorrhage Due to Rupture of Jejunal Artery Aneurysm in Behcet Disease: Case Report and Literature Review. Medicine (Baltimore) 94(45): e1979.

13. Davis PG, Fordham JN, Kirwan JR, Barnes C, Dinning W (1984) The pathergy test and Behqet's syndrome in Britain. Ann Rheum Dis 43(1): 70-73.

14. Fowler TJ, Hempson DJ, Nussy AM, Small M (1968) Behqet's syndrome with neurological manifestation in two sisters. BMJ 2(5603): 473.

15. Cornelis F, Sigal-Nahum M, Gaulier A (1989) Behqet's disease with severe cutaneous necrotizing vasculitis: Response to plasma exchangereport of a case. J Am Acad Dermatol 21: 576-579.

16. Rustin MH, Gilkes JJ, Robinson TW (1990) Pyoderma gangrenosa associated with Behqet's disease: Treatment with thalidomide. J Am Acad Dermatol 23: 941-994.

17. Saleh Z, Arayssi T (2014) Update on the therapy of Behçet disease. Ther Adv Chronic Dis 5(3): 112-134.

18. Enoch BA, Castillo-Olivares JL, Khoo TC, Grainger RG, Henry L (1968) Major vascular complications in Behçet's syndrome. Postgrad Med J 44(512): 453-459.

19. Chafa O, Fisher AM, Meriane F, Sternberg C, Otmani F, et al. (1992) Behqet's syndrome associated with protein $\mathrm{S}$ deficiency. Thromb Haemost 67(1): 1-3.

20. Shento NM, Ghosh K, Abdul Kader B, al Assad HS (1992) Extensive venous thrombosis in a case of Behqet's disease associated with heterozygous protein C deficiency [letter]. Thromb Haemost 67(2): 283.

21. Aitchison R, Chu P, Cater DR (1989) Defective fibronolysis in Beh§et's syndrome: Significance and possible mechanisms. Ann Rheum Dis 48: 590-593.

22. Kage H, Goto Y, Amano Y, Kosuke Makita, Hideaki Isago, et al. (2016) Development of Pulmonary Artery Aneurysms Due to Behçet's Disease and Resolution after Treatment. Intern Med 55(22): 3337-3340.

23. Shen C, Li W, Zhang Y, Qingle Li, Yang Jiao, et al. (2016) Outcomes of surgery for patients with Behcet's disease causing aortic pseudoaneurysm: a shift from open surgery to endovascular repair. Clinics (Sao Paulo) 71(6): 302-310.

24. Brudbury AW, Milne AA, Murie JA (1994) Surgical aspects of Behcet's disease. Br J Surg 81: 1712-1721.

25. Tuizi H, Sayin A, Karaozbek Y (1993) Peripheral aneurysms in Behcet's disease. Cardiovasc Surg 1: 220-224.

26. Hassan H, Al-Orainy I, Rabee H, Daif A (2004) Imaging findings of neuroBehcet disease. Neurosciences 9(3): 180-185. 